Review

\title{
Revisiting the Challenges in Fabricating Uniform Coatings with Polyfunctional Molecules on High Surface Energy Materials
}

\author{
Stephanie Oyola-Reynoso ${ }^{1}$, Zhengjia Wang ${ }^{1}$, Jiahao Chen ${ }^{1,2}$, Simge Çınar ${ }^{1}$, Boyce Chang ${ }^{1}$ \\ and Martin Thuo ${ }^{1,2,3, *}$
}

1 Department of Materials Science and Engineering, Iowa State University, 2220 Hoover Hall, Ames, IA 50011, USA; E-Mails: so1@iastate.edu (S.O.-R.); zhengjia@iastate.edu (Z.W.); jiahao@iastate.edu (J.C.); scinar@iastate.edu (S.Ç.); boyce@iastate.edu (B.C.)

2 Micro-Electronic Research Center, Iowa State University, 133 Applied Sciences Complex I, 1925 Scholl Road, Ames, IA 50011, USA

3 Biopolymer and Biocomposites Research Team, Center for Bioplastics and Biocomposites, Iowa State University, 1041 Food Sciences Building, Ames, IA 50011, USA

* Author to whom correspondence should be addressed; E-Mail: mthuo@iastate.edu; Tel.: +1-515-294-8581.

Academic Editor: James Tsoi

Received: 1 October 2015 / Accepted: 4 December 2015 / Published: 18 December 2015

\begin{abstract}
Modifying the chemistry of a surface has been widely used to influence interfacial properties of a material or nature of interaction between two materials. This article provides an overview on the role of polyfunctional molecules, specifically silanes, in surface modification of polar surfaces (bearing soft nucleophiles). An emphasis on the mechanism of the reaction in the presence of adsorbed water, where the modifying reagents are hydrolysable, is discussed. To highlight the complexity of the reaction, modification of paper with trichlorosilanes is highlighted. Preparation of hydrophobic cellulosic paper, and structure-property relations under different treatment conditions is used to highlight that a monolayer is not always formed during the surface modification. Gel-formation via step-growth polymerization suggests that at the right monomer:adsorbed water ratio, a monolayer will not form but rather self-assembly driven particle formation will occur leading to a textured surface. The review highlights recent work indicating that the focus on monolayer formation, is at the very least, not always the case but gel formation, with concomitant self-assembly, might be the culprit in understanding challenges associated with the use of polyfunctional molecules in surface modification.
\end{abstract}


Keywords: silane; surface science; coatings; monolayers; polymer gel; surface water

\section{Introduction}

Molecules containing two or more reactive moieties are defined as polyfunctional ("polyvalent" in gel formation discussions) molecules. The properties of such molecules are typically dictated by their functional groups, and as such, are expected to react stochastically with multiple units a singly functionalized reagent, except in presence of a site-selective catalyst. In the presence of polyfunctional reagent(s), the stochastic nature of the reaction between the two species can yield different products depending on the ratio of both reagents [1-11]. Silane reagents in the form of $\mathrm{X}_{n}$-Si-(R) ${ }_{4-n}$, (where; $\mathrm{R}=$ alkyl/organic fragment, $\mathrm{X}=$ halide or other reactive species; Figure 1) are examples of polyfunctional molecules bonding reaction commonly used for surface modification [12-14]. In addition, organofunctional silanes behave as hybrids between silica and organic materials making them one of the best choice for coupling agents [15-23]. Silanes, as surface modifying reagents, are known to readily chemisorb on most materials to either form entangled covalent networks, cross-linked monolayers or, in the case of silanols on hydroxylated surfaces, monolayers derived from hydrogen-bonding networks (Figure 1).

a)

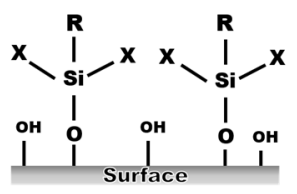

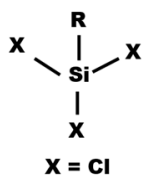

$\mathbf{X}=\mathbf{C}$ b)

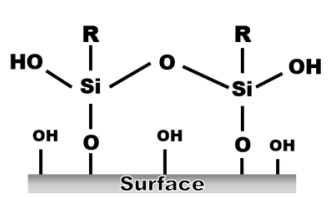

c)

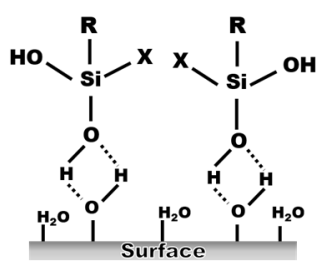

Figure 1. Schematics examples of the different commonly used siloxane reactions reported in literature [12-14]. Reaction of a hydroxylated surface with an alkyl silane to either form a covalent bond $(\mathbf{a}, \mathbf{b})$ or, in the case of siloxanes, form a hydrogen bond (c).

\subsection{Surface Modification}

The surface chemistry of a material plays an essential role in determining its properties since these surfaces dictate intra- and inter-material interactions and/or interfacial stimuli response to the material - the basis of many smart materials and materials applications. Surface modification, which can be achieved by etching [24], chemical treatment [25,26], or physical treatment [27,28], provides the opportunities to tune optical, electrical, [29-35] and interfacial [36-38] material properties. Owing to the availability of reactive groups, mostly soft nucleophiles or electrophiles, most polymer surfaces can be readily modified through chemical grafting by simply exposing the material to an appropriate reagent(s) [1,7-9,14,15,17-22,39-52].

The use of silanes for surface modification dates back to the 1940s when it was discovered that the use of materials like polyester and urea to manufacture silicate glass-reinforced composites produced 
unreliable composites due to their sensitivity to humid environments [18]. This led to the use of silanes that are capable of changing the surface affinity to water vapor [18] without affecting mechanical properties of the materials. Ever since their discovery, silanes have been widely used in the manufacture of composites, and also for various coatings applications [53,54]. Trialkoxysilanes are the most commonly used coupling agents in composite manufacturing due to favorable compatibility with many polymer resins [19]. Besides being chemisorbed, silanes can also interact with polymeric surfaces like cellulose through hydrogen bonding. Abdelmouleh et al. reported the use of silane coupling agents to modify cellulose in an ethanol/water mixture [17]. They then quantitatively studied the behavior of cellulose fibers upon treatment with the silanes and found that, as expected for hydrolyzed silanes, washable physisorbed layers are formed. Upon thermal treatment of the reaction mixture, a chemisorbed (non-washable) coating was realized, and they suggested that temperature was an essential component for the chemical reaction to occur (Figure 2a-d) [17]. Further advances were reported by Pallandre et al. [40] describing the fabrication of flat surfaces by nanoscale chemical patterns obtained from lithography and gas phase silane monolayers (Figure 2e,f). Pallandre et al. reported a method where lithographically generated masks were used to define binary nano-patterns on silicon wafers (Figure 2e,f). In this report, Pallandre et al. indicate that a monolayer was being formed on regions that were not masked, as seen in Figure 2e,f, for the Atomic Force Microscopy analysis of the silane treated silicon wafers. Other studies have followed with different indirect observations, and so far the use of polyfunctional silanes, especially the trichlorosilanes, is often seen as complicated.
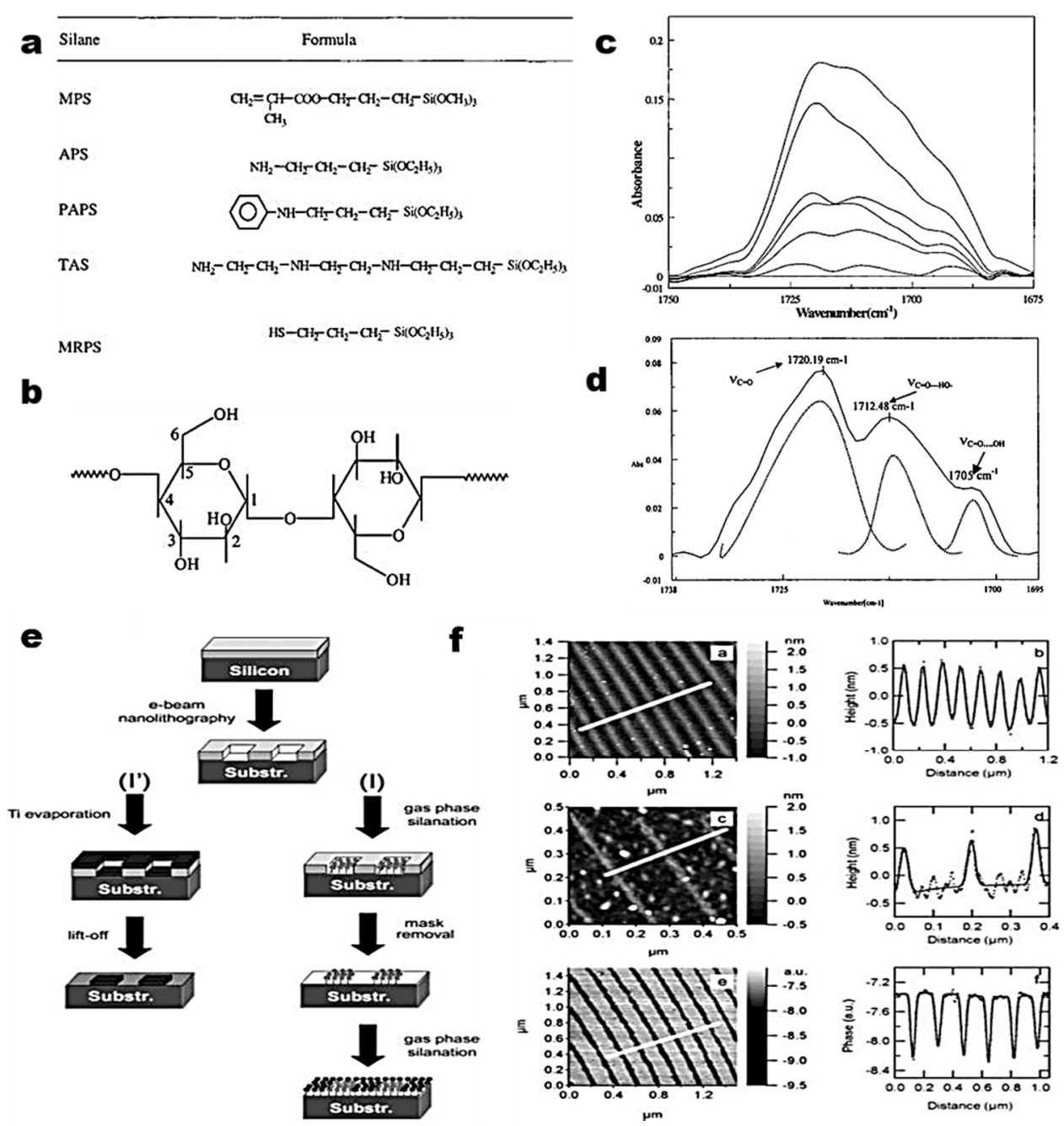
Figure 2. (a-d) Silane coupling agents used in the study of cellulose interactions with silanes and evolution study of the $\mathrm{C}=\mathrm{O}$ peaks of sample treated with different silane concentrations with respect of the ethanol/water mixture [17] (Reprinted from ref. [17], Copyright Langmuir 2002); (e,f) Schematic of the path taken to produce nano patterned substrate and surface analysis of silane treated silicon wafers [40] (Reprinted from ref. [40], Copyright American Chemical Society 2004).

\subsection{Surface Modification by Silane Treatment on Polymers}

Surface modifications by silane treatments have been recently used to introduce chemically reactive functional groups onto polymer substrates $[11,24,55,56]$. Careful selection of the head groups and alkyl chain components can result in desirable wettability properties, adhesion characteristic, and surface composition. Silane surface treatment can generate self-cleaning surfaces and decrease surface contaminants [57-59]. The alkyl groups of the organosilicon are, sometimes, hydrolyzed to form silanol-containing species, via a four-step reaction: (1) hydrolysis of the head groups, (2) condensation/ oligomerization of the hydroxylated products, (3) hydrogen bonding between the oligomers and surfacebound water, and, (4) covalent linkages formed with the substrate during drying or curing [60]. The degree of polymerization significantly depends on the amount of water, which may corresponding to; (a) added water, (b) water present in the substrate, or (c) adventitiously adsorbed water (more details on surface water is discussed in the following sections) [61]. Alkyl silanes reacts with polymers, leading to the attachment of the trialkoxylsilane group onto the polymer backbone. The silane is then available for further reaction with moisture to crosslink and, therefore, stabilize into a three dimensional structure $[14,60]$. This interesting feature finds many useful applications for paints, coatings, biological devices, electronics and reinforcement of thermosets. Wong et al. reported a bioinspired self-repairing slippery surfaces by pressure-stable Omniphobic materials [62], and this work was extended for use with paper by Glavan et al. Wong and coworkers described the phenomenon as a formation of a uniform silane monolayer attached to the surface of cellulose, based on the reactivity of silane with hydroxyl groups. Their work was expanded to the use of hydrophobic paper infused with perfluoropropylether to create slippery surfaces via SLIPS (Slippery Liquid Infused Porous Surfaces) (Figure 3).

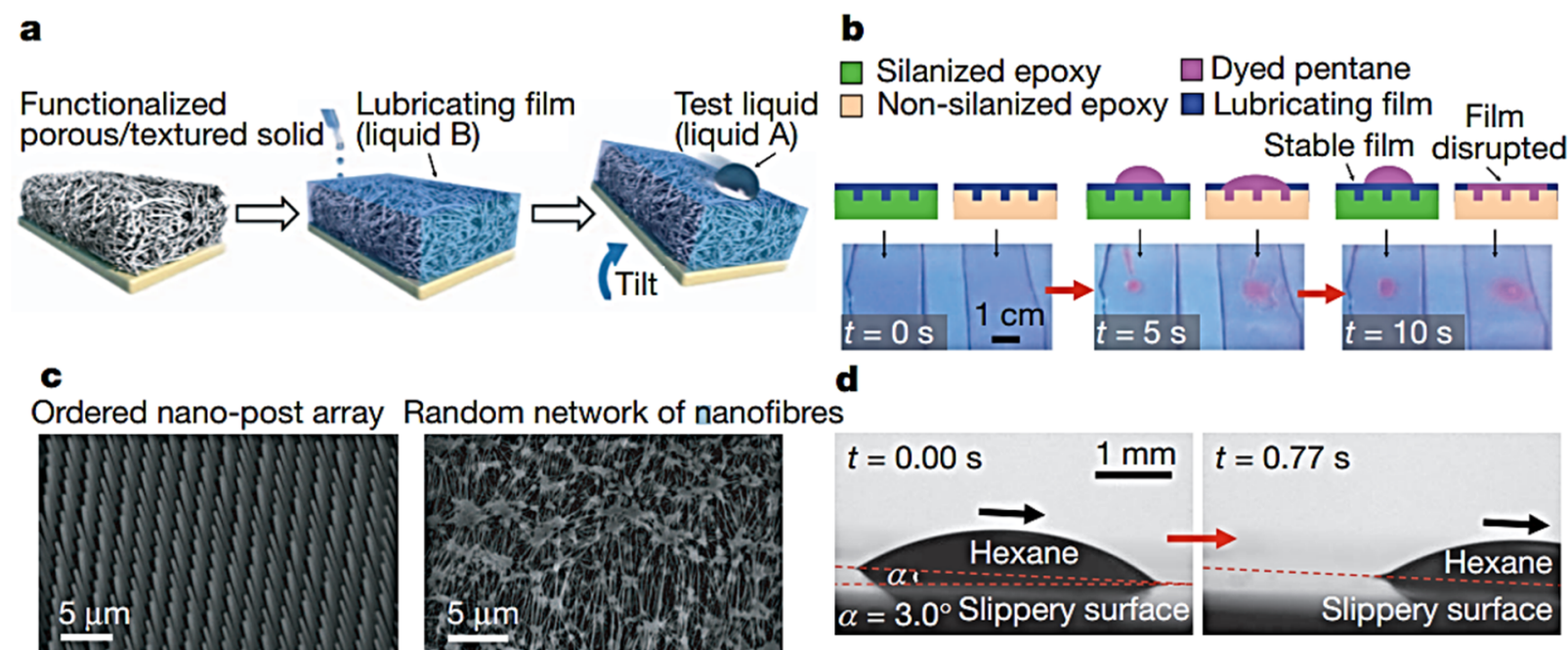


Figure 3. (a) Representation of cellulosic material response upon lubricant surface treatment; (b) chemical surface treatment of SLIPS; (c) SEM high magnification images of surface texturing after treatment; and (d) hysteresis contact angle for hexadecane on SLIPS surfaces [62]. (Reprinted with permission from ref. [62]; Copyright Nature 2011).

\section{Silanes in Textile Applications}

Surface modification techniques have been widely employed for improving textile functional performances, such as softness, dyeability, wettability, lubricity, anti-winkle and anti-microbial properties for decades [49,63-70]. Surface modification techniques in textiles are divided into: chemical, mechanical, or combined methods. Normally, such modification is accomplished through the padding or the exhaust process, spraying, plasma treatment, and layer-by-layer assembly [68,71-73]. Silane chemistry has attracted particular interests in textiles surface modification especially for hydrophobic property. In 1945, the first patent on textile surface treatment was filed by Norton [74]. This patent described the use of alkyl silane to modify the surface wetting properties of textiles [74]. Since then several methods have been reported for the application of silanes in textiles for surface modification [44,49]. A common technique used is the spraying technique due to its simplicity and high water contact angles upon treatment. Spraying technique involves silanes dissolved in organic solvents or solvent/water mixtures, then the prepared solution is directly sprayed onto the fibers [75]. For natural fiber based fabrics, however, spraying only results in a surface coating as the inside of the cell walls remains untreated [20], mainly due to the need for higher diffusion time for the silane to penetrate into the dense fiber cell walls. Other methods reported involves immersion of the textiles in a silane mixture for the sol-gel process [76]. This method requires post treatment processing like padding and/or heat treatment. In this process, silane mixtures are transferred from aqueous solution phase to the fabric surface, both the fiber surfaces and cell walls are modified with the silanes sol-gel solution. This technique although simple, it takes time for the hydrolysis and condensation to proceed to an appreciable degree. It has been hypothesized that the penetration of silane into the cell walls is influenced by the molecular size of silane as well as the aging of the hydrolyzed silane solution. In this case, diffusion of silanes into the cell walls will be limited or prevented entirely if the molecular size of the silane increases due to fast condensation of silanols [20]. The treatment of fiber walls can change the properties of the cell walls, and as a result the performance or properties - like the whiteness (e.g., cotton color due to heat treatment) and strength of natural fabrics. Zhou et al. reported a super-hydrophobic polyester fabric made of a crosslinked polydimethylsiloxane elastomer containing silica nanoparticles and fluorinated alkyl silane by dip coating and curing (Figure 4a) [44]. They demonstrated that the treated fabric has remarkable durability against repeated machine washes and excellent stain resistance (Figure 4b,c). Properties imparted to textiles using silane solution include water repellence, soil/dirt resistance, wrinkle resistance, anti-bacteria, anti-static, UV-protection, flame retardation, and improvement of dyeability, among others [21,49,51,65,77]. The main problem, however, is that most of these methods do not offer permanent modification and the fabric tends to lose the new property upon laundering or use. 

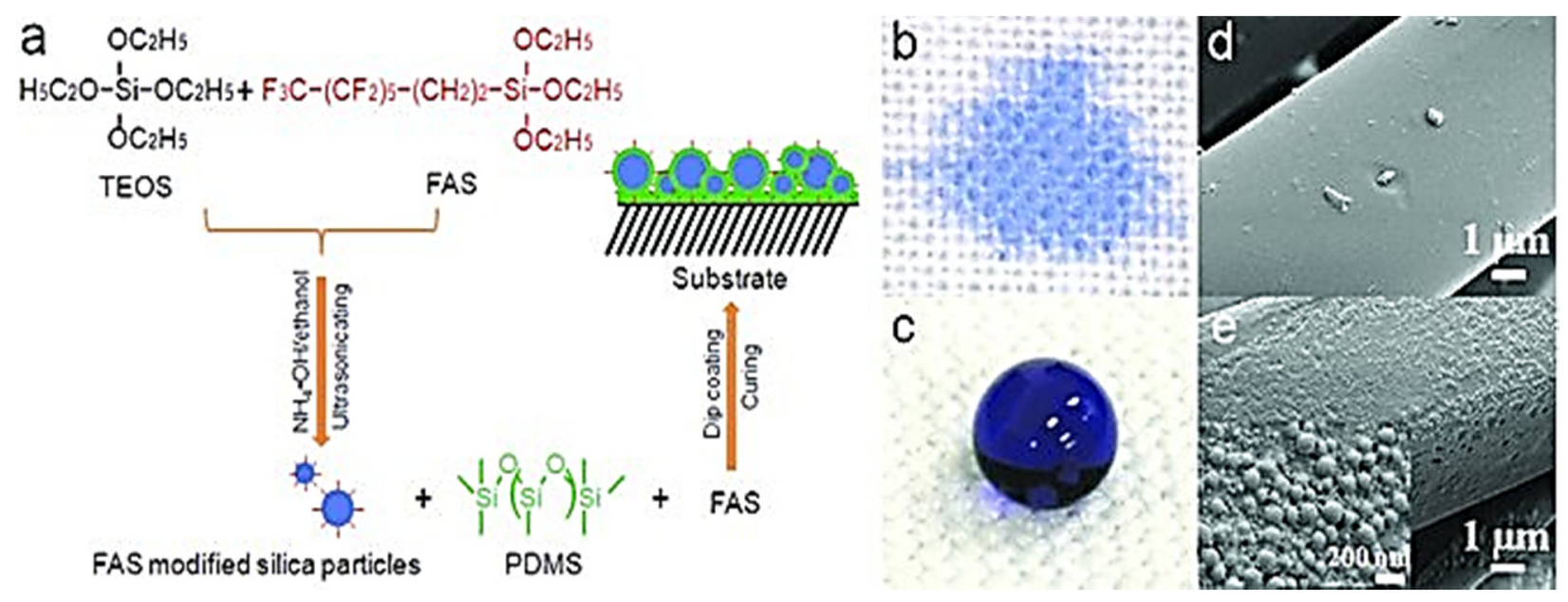

Figure 4. (a) Procedure to prepare the silica particles and super-hydrophobic fabrics; (b) the picture of water droplet $(10 \mu \mathrm{L}$ each) on the untreated polyester fabrics; (c) the picture of water droplet on treated polyester fabrics; (d) SEM image of pure polyester fabric; and (e) SEM image of silica/PDMS/FAS treated fabric[44]. Reprinted with permission from ref. [44]; Copyright Wiley Online Library 2012.

\section{Silane in Polymer Chemistry}

\subsection{Biomimetic}

Nature provides a library of methods for surface engineering, for example, the mesmerizing surfaces on beetles, plants, and lizards living in xeric environments. These interesting properties serve as inspiration to create and improve material surfaces. Advances in surface modification have led to the formation of affordable and reproducible devices that can be applied in areas like biology, electronics, adhesives, and, coatings. The Stenocara gracilipe, also known as the desert Namib Beetle, has the ability to harvest water from fog in otherwise dry, desert, environments. This behavior is possible due to the microstructure of the beetles wings, which has hydrophilic bumps that allows the adhesion of water while the valleys are hydrophobic allowing flow of the collected water into its mouth. Reported works on surface modification indicates that silanes are prone to form a monolayer when exposed to cellulosic surfaces. We, however, recently showed that by tuning the ratio of the surface area, hence the amount of surface adsorbed water, relative to vapor phase silane, the reaction of paper with trichlorosilanes does not give monolayers but rather leads to formation of chemisorbed polysiloxane gel particles [78]. We validated the formation of oligomer gel particles, which occurs through reaction of silane with bound water on cellulose by characterizing the resulting new texture on the surface treated paper [78]. We further support the possibility of gel-particle formation through the gel-formation coefficient (Equation (1)) where the ratio of the two polyvalent reactants, in this case water (bivalent) and trichlorosilane (trivalent) are pre-disposed to gel given the right concentration ranges. Reaction of the trichlorosilane and water should lead to a polymeric material through step-growth type mechanism, hence, at low-degrees of polymerization, the formed material would be too small to image (Carother's paradox). The presence of a hydrophobic tail on the silane should induce micellar-like assembly of the reacting species leading to, in an ideal case, spherical structures (due to surface energy minimization) on the surface of the material. This assembly process should also depend on the hydrophobicity of the tail, 
and we showed that, in fact, perfluorinated silanes form particles on the surface of the paper faster than the non-fluorinated analogs.

$$
\alpha=\frac{r p_{A}^{2} p}{1-r p_{A}^{2}(1-p)}=\frac{p_{B}^{2}}{r-p_{B}^{2}(1-p)}
$$

The branching coefficient (Equation (1)) takes into account the following parameters for any two monomers containing reactive moiety " $\mathrm{A}$ " and reactive moiety " $\mathrm{B}$ ": (i) ratio of the number of reactive moieties A to B (r), (ii) probability of finding either the $\mathrm{A}\left(p_{A}^{2}\right)$ or $\mathrm{B}\left(p_{B}^{2}\right)$ moieties in the polymerized material, and (iii) the probability of finding an unreacted group $(1-p)$ [79].

\subsection{Cellulose Surface Chemistry}

Cellulose chemistry has been studied for many years and yet until recently, the surface modification of cellulose was not fully understood. We reported a surface polymerization process using perfluorinated alkyl silane reagent on a high grammage $\left(\sim 165.74 \mathrm{~g} / \mathrm{m}^{2}\right)$ paper [37]. Surface analysis of treated paper with Scanning Electron Microscopy (SEM) showed that particles (Figure 5d) were formed on the surface. Paper was treated with a trichloro $(1 \mathrm{H}, 1 \mathrm{H}, 2 \mathrm{H}, 2 \mathrm{H}$ perfluorooctyl)silane with or without heated reaction allowed to proceed for different reaction times. Surface particulates were seen only after vapor deposition on a high grammage paper (Figure 5c). These particulates increased in size, as well as their surface coverage, with increased reaction time (Figure 5d). Wetting properties of treated paper varied with size and/or surface coverage of the surfaces by the gel particles as shown in the trend of the contact angle for different reaction times performed with fluorinated organosilane with a highest value of $120^{\circ}$ $\pm 2^{\circ}, n=10$ (Figure 5f).

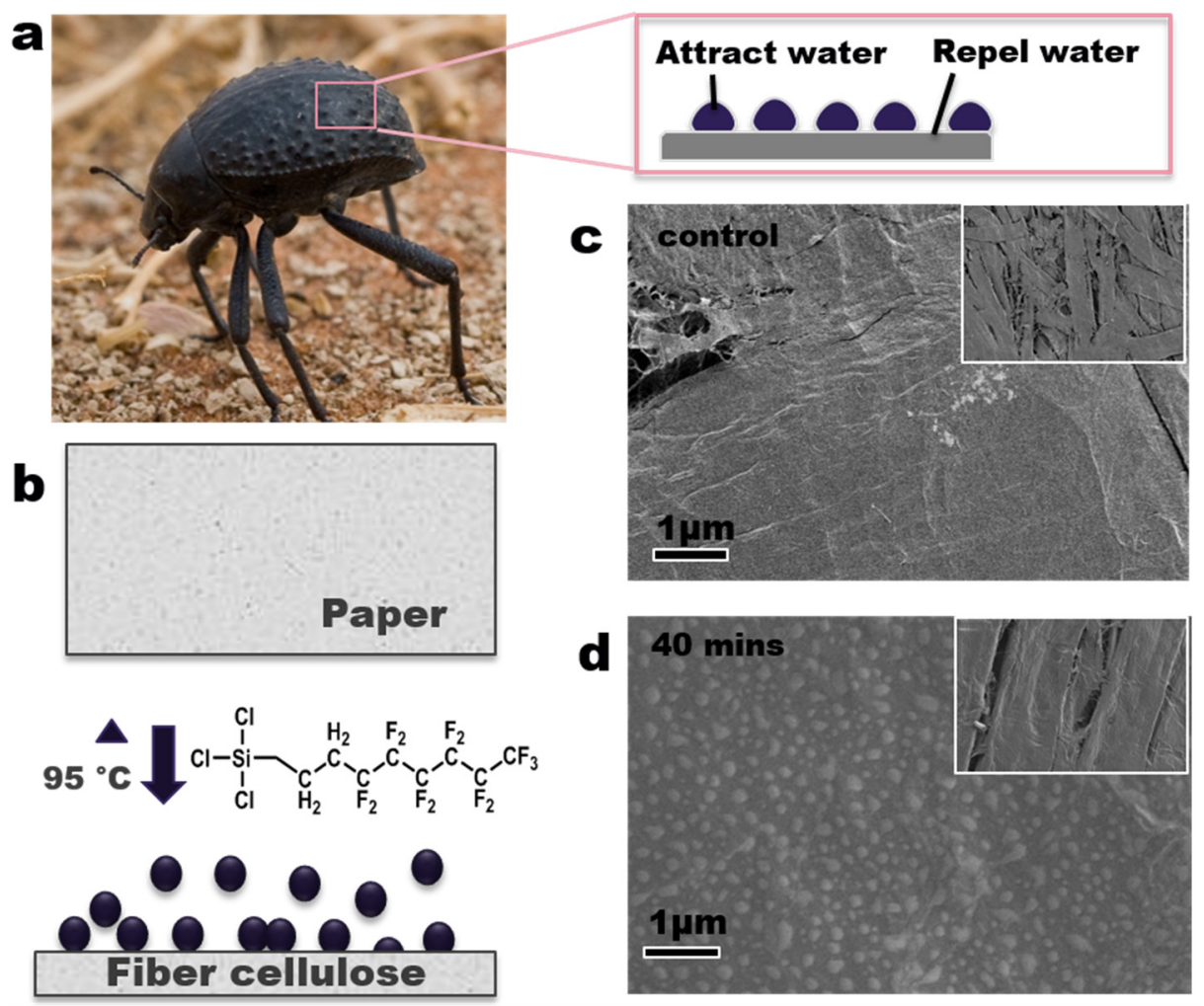

Figure 5. Cont. 

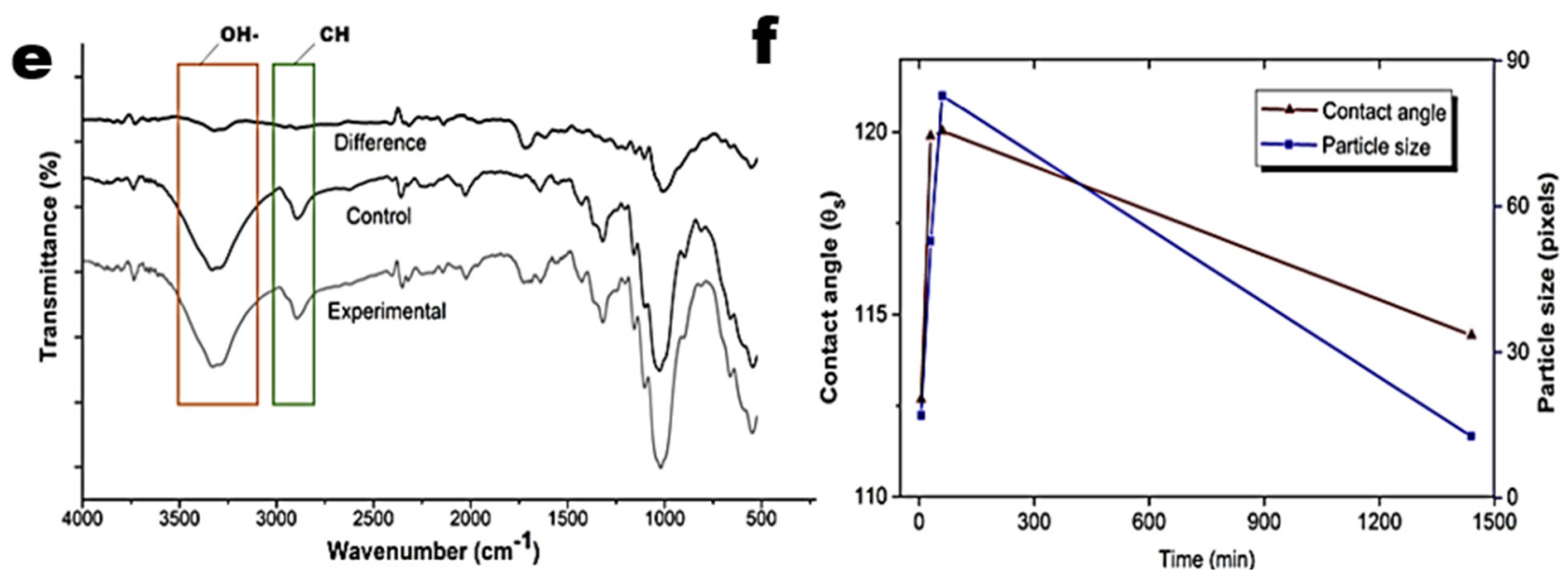

Figure 5. (a) Representation of dessert Namib beetle and its wing micro structure; (b) schematics of silane gas-phase treatment; and (c) native high grammage $\left(\sim 165.74 \mathrm{~g} / \mathrm{m}^{2}\right)$ paper image before silanization. (d) Upon salinizing the surface of the high grammage paper, small particles are seen when reacting the surface with a fluorinated silane; (e) Infrared analysis of control and silane treated paper; (f) Contact angles and particle size of treated paper [78].

\subsection{Bound Water}

Intra- and inter-molecular interactions determine the state and behavior of materials and systems. Among its unusual, if not unique, properties of water, the ability to form hydrogen bonds makes water one of the most effective and common solvents in nature. As water comes in contact with surfaces of hydrophilic or polar materials, the structure of water at the interface differs from the bulk, and interferes with mechanical and chemical behaviors of surfaces and materials (Figure 6a). Therefore, the interfaces in exposure to water or humidity gain significant importance not only to control mechanical and chemical behavior of surface and material, but also to explore new applications [6,54,80-86]. Even though understanding of this bound water layer remains incomplete, it has been shown that the changes in structure of water are dominated by hydrogen bond networks and its dynamic evolution [82,87-89].

The first few layers of water that are chemically adsorbed on the surface of material are known as freezing bound water because its configuration and behavior resembles ice, especially due to restricted motion of the molecules [87,90-92]. Following layers of water associated with this first bound water layer is called non-freezing bound water and behaves distinctly different from the bulk water even though its intermolecular interactions are weaker than the ones of non-freezing water (Figure 6a).

It is therefore clear that not only is the bound water layers, but also the surface of material(s), affected by any hydrophilic and/or hydrophilic interactions at the interface. The extent of the influence both on the bound water and the materials' surface, however, depends on the strength of interaction, and therefore on the properties of the material [93]. In oxide and hydroxide systems, strong hydrogen bonds between hydroxyl and oxygen atoms between the oxide surface and the water molecules significantly interfere with the surface structure because water molecules penetrate into the structure and, in some cases, result in swelling at the surface $[83,94,95]$. Similarly, surface of polymers with charge or functional groups capable of hydrogen bonding (e.g., hydroxyls, carboxyls, and, amines) are affected by 
the presence of water and, therefore, forms strong bound water layers $[82,91,92,96]$. Strong affinity to hydrogen bonding, hence favorable secondary interactions, draws water towards the inside/core of a porous/fibrous structure, weakening the interactions between polymer chains leading to swelling. Plasticizing effect of bound waters often leads to lowering of the Young's modulus (mechanical strength) of polymeric materials $[3,97,98]$. Fibrous polymeric materials like cellulose contain multiple layers of water, which interact with the silane surface treatment before it allows the external molecules to be attached the cellulose fibers, as shown in Figure 6b. In some hydrogels, however, bound water layer let to higher Young's moduli (stronger mechanical properties) as compared to the ones filled only with bulk water [6]. In another example, the presence of bound water on surfaces was reported to prevent fouling of materials, thus used to promote antifouling properties of surfaces $[80,84]$.
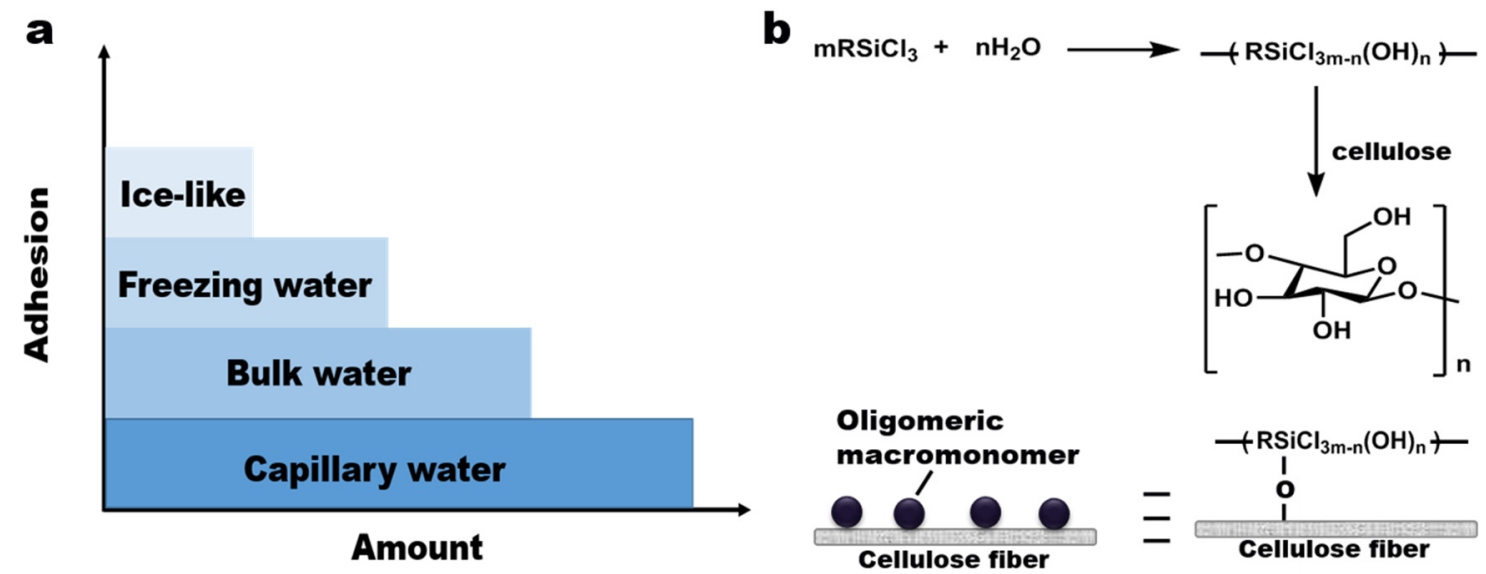

Figure 6. (a) Schematic of surface bound water of cellulose based on the water adhesion and water amount at the surface of cellulose; (b) Hypothesized reaction pathway of silane to water and cellulose to form a macro-monomer [99].

The bound water content may be reduced in vacuum or high temperature environment and increased by exposing to water vapor, aqueous environment or humidity. Even though the bound water content on surfaces may be tuned to some extent, its effect should be considered during any step of processing or application because of adsorption of adventitious water molecules from ambient conditions.

\subsection{Surface Roughness}

Vapor deposition of silane introduces a roughness level that significantly affects wetting properties $[61,100]$. The hydrophobicity (or in general non-wettability) of a solid surface is heavily dependent on the surface energy and the roughness (micro-structure) of the surfaces. The widely used Young's equation indicates that the contact angle $(\theta)$ of a droplet is a function of three interfacial energies, as shown in Equation (2), namely solid-liquid $\left(\gamma_{\mathrm{SL}}\right)$, solid-vapor $\left(\gamma_{\mathrm{SV}}\right)$, and liquid-vapor $\left(\gamma_{\mathrm{LV}}\right)$.

$$
\cos (\theta)=\frac{\gamma_{S V}-\gamma_{S L}}{\gamma_{L V}}
$$

The Young's equation is, however, only applicable to contact angle of droplet on flat, chemically stable, and, homogeneous surfaces. The interaction between a rough surface and probe liquid leads to misinterpretations based on the Young's equation since, in some cases, the $\gamma_{S L}$ is only a fraction of the 
total footprint of the droplet. A majority of surfaces are not ideally flat, and may offer only a fraction (higher than or less than predicted) of the solid material to be in contact with the probe liquid in the so-called Cassie (less contact area with the material) or Wenzel (higher contact area with the material) state (Figure 7). By modifying the Young's equation, Wenzel proposed a new model (Equation (3)) by introducing the roughness factor, $(r)$, defined as the ratio of the actual area of the surface in contact with the liquid to its geometrically projected area.

$$
\cos \left(\theta^{\prime}\right)=\frac{r\left(\gamma_{S V}-\gamma_{S L}\right)}{\gamma_{L V}}=r \cos (\theta)
$$

According to Wenzel's theory, (i) reducing surface energy ( $\left.\gamma_{\mathrm{SV}}\right)$, and (ii) increasing the roughness of a hydrophobic surface will increase the hydrophobicity (contact angle) of a surface. As discussed before, polyfunctional molecules for cellulose surface modification with lowest surface energy are the $-\mathrm{CF}_{3}$ groups, which can provide large water contact angle $\left(120^{\circ}-147^{\circ}\right)[58,78,101]$. The roughness factor $r$ is defined as:

$$
r=1+\frac{S_{r d}}{100}
$$

where $S_{\text {rd }}$ is the roughness parameter. Since $r$ is always $>1$, increasing roughness of hydrophilic surface $\left(\theta<90^{\circ}\right)$ leads to increased hydrophilicity while increasing roughness for hydrophobic surfaces $\left(\theta>90^{\circ}\right)$ leads to increased hydrophobicity. Incorporating increased roughness and low surface energy, higher hydrophobicity of surface can thus be achieved. Most of the methods to alter the surface chemistry, however, also modify both the surface topography and/or morphology In 2013, Song and Rojas gave a good overview of current methods to make hydrophobic paper[100], which include: (1) Chemical grafting to decreasing the surface energy by modifying the surface chemistry and change the surface topography simultaneously; (2) micro/nano-particle by loading the nano particles to the cellulose fiber to change the surface topography and change the surface energy post-treatment; (3) sol-gel processing; (4) electrospinning and electrospraying, and (5) using nano cellulose and cellulose composite to fabricate papers.

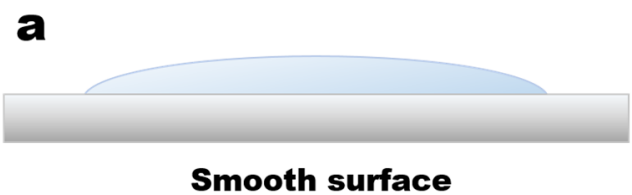

Smooth surface

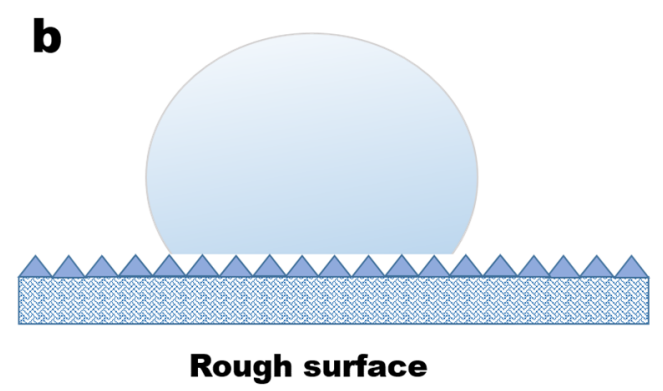

Figure 7. Schematic of water droplet behavior on a smooth (a) and rough surface (b). 


\section{Summary}

An understanding of the role of polyfunctional molecules, here silane, in engineering surface properties is reviewed. With high demand of affordable devices and/or materials, paper is expected to play an essential role in low cost device manufacturing, with surface engineering of cellulose being central to that objective. Methods involving the use of polyfunctional molecules in surface modification of cellulose have high potential impact in low cost surface treatment. Potential applications from surface modification with polyfunctional molecules include bio-analytical tools, medical devices, electronics, and adhesion, among others. Covalent bond formation between the silane component and the matrix of the interface material, allows the molecule to form networks producing "micelle like" particulates on the materials surface with concomitant change in wetting properties.

\section{Acknowledgments}

This work was supported by Iowa State University through startup funds. M.T. acknowledges support through a Black and Veatch Building a World of Difference Faculty Fellowship from Iowa State University. M.T. and J.C. were supported in part by a Catron fellowship from the Engineering Research Institute.

\section{Author Contributions}

The review was written through contributions of all authors under the direction from Martin Thuo.

\section{Conflicts of Interest}

The authors declare no conflict of interest.

\section{References}

1. Maddox, A.F.; Matisons, J.G.; Singh, M.P.; Zazyczny, J.; Arkles, B. Cyclic azasilanes: A kinetic approach to rapid silane surface modification. In Proceedings of the 249th ACS National Meeting \& Exposition, Denver, CO, USA, 22-26 March 2015.

2. Pouxviel, J.C.; Boilot, J.P. Kinetic simulations and mechanisms of the sol-gel polymerization. J. Non-Cryst. Solids 1987, 94, 374-386.

3. Yakimets, I.; Wellner, N.; Smith, A.C.; Wilson, R.H.; Farhat, I.; Mitchell, J. Mechanical properties with respect to water content of gelatin films in glassy state. Polymer 2005, 46, 12577-12585.

4. Shea, K.J.; Loy, D.A. A mechanistic investigation of gelation. The sol-gel polymerization of precursors to bridged polysilsesquioxanes. Acc. Chem. Res. 2001, 34, 707-716.

5. Mauritz, K.A. Organic-inorganic hybrid materials: Perfluorinated ionomers as sol-gel polymerization templates for inorganic alkoxides. Mater. Sci. Eng. C 1998, 6, 121-133.

6. Pasqui, D.; De Cagna, M.; Barbucci, R. Polysaccharide-based hydrogels: The key role of water in affecting mechanical properties. Polymers 2012, 4, 1517-1534.

7. Textor, T.; Mahltig, B. A sol-gel based surface treatment for preparation of water repellent anti-static textiles. Appl. Surf. Sci. 2010, 256, 1668-1674. 
8. Kiraz, N.; Burunkaya, E.; Kesmez, O.; Asilturk, M.; Camurlu, H.E.; Arpac, E. Sol-gel synthesis of 3-(triethoxysilyl)propylsuccinicanhydride containing fluorinated silane for hydrophobic surface applications. J. Sol-Gel Sci. Technol. 2010, 56, 157-166.

9. Burunkaya, E.; Kiraz, N.; Kesmez, O.; Asilturk, M.; Camurlu, H.E.; Arpac, E. Sol-gel synthesis of IPTES and D10H consisting fluorinated silane system for hydrophobic applications. J. Sol-Gel Sci. Technol. 2010, 56, 99-106.

10. Kaufman, V.R.; Avnir, D.; Pines-Rojanski, D.; Huppert, D. Water consumption during the early stages of the sol-gel tetramethylorthosilicate polymerization as probed by excited state proton transfer. J. Non-Cryst. Solids 1988, 99, 379-386.

11. Tshabalala, M.A.; Sung, L.-P. Wood surface modification by in-situ sol-gel deposition of hybrid inorganic-organic thin films. J. Coat. Tech. Res. 2007, 4, 483-490.

12. Goerl, U.; Hunsche, A.; Mueller, A.; Koban, H.G. Investigations into the silica/silane reaction system. Rubber Chem Technol. 1997, 70, 608-623.

13. Chan, T.-H.; Melnyk, A. Kinetics and mechanism of the sulfoxide-silane reaction. J. Am. Chem. Soc. 1970, 92, 3718-3722.

14. James, E.M. Overview of Siloxane Polymers. In Silicones and Silicone-Modified Materials; American Chemical Society: Washington, DC, USA, 2000; Volume 729, pp. 1-10.

15. Nakatani, H.; Iwakura, K.; Miyazaki, K.; Okazaki, N.; Terano, M. Effect of chemical structure of silane coupling agent on interface adhesion properties of syndiotactic polypropylene/cellulose composite. J. Appl. Polym. Sci. 2011, 119, 1732-1741.

16. Ukaji, E.; Furusawa, T.; Sato, M.; Suzuki, N. The effect of surface modification with silane coupling agent on suppressing the photo-catalytic activity of fine $\mathrm{TiO}_{2}$ particles as inorganic UV filter. Appl. Surf. Sci. 2007, 254, 563-569.

17. Abdelmouleh, M.; Boufi, S.; ben Salah, A.; Belgacem, M.N.; Gandini, A. Interaction of silane coupling agents with cellulose. Langmuir 2002, 18, 3203-3208.

18. Plueddemann, E.P. Silane Coupling Agents, 2nd ed.; Springer US: New York, NY, USA, 1991.

19. Matisons, J.G. Silane coupling agents and glass fibre surfaces: A perspective. In Silanes and Other Coupling Agents; CRC Press: Boca Raton, FL, USA, 2009; Volume 5, pp. 1-24.

20. Xie, Y.; Hill, C.A.S.; Xiao, Z.; Militz, H.; Mai, C. Silane coupling agents used for natural fiber/polymer composites: A review. Compos. A Appl. Sci. Manuf. 2010, 41, 806-819.

21. Mittal, K.L. Silanes and Other Coupling Agents; CRC Press: Boca Raton, FL, USA, 2007; Volume 4.

22. Salon, M.-C.B.; Gerbaud, G.; Abdelmouleh, M.; Bruzzese, C.; Boufi, S.; Belgacem, M.N. Studies of interactions between silane coupling agents and cellulose fibers with liquid and solid-state NMR. Magn. Reson. Chem. 2007, 45, 473-483.

23. Sabzi, M.; Mirabedini, S.M.; Zohuriaan-Mehr, J.; Atai, M. Surface modification of $\mathrm{TiO}_{2}$ nano-particles with silane coupling agent and investigation of its effect on the properties of polyurethane composite coating. Prog. Org. Coat. 2009, 65, 222-228.

24. Liu, Y.; Yin, X.; Zhang, J.; Wang, Y.; Han, Z.; Ren, L. Biomimetic hydrophobic surface fabricated by chemical etching method from hierarchically structured magnesium alloy substrate. Appl. Surf. Sci. 2013, 280, 845-849. 
25. Croll, T.I.; O’Connor, A.J.; Stevens, G.W.; Cooper-White, J.J. Controllable surface modification of poly(lactic-co-glycolic acid) (PLGA) by hydrolysis or aminolysis I: Physical, chemical, and theoretical aspects. Biomacromolecules 2004, 5, 463-473.

26. Chan, C.M.; Ko, T.M.; Hiraoka, H. Polymer surface modification by plasmas and photons. Surf. Sci. Rep. 1996, 24, 1-54.

27. Chitnis, G.; Ding, Z.; Chang, C.-L.; Savran Cagri, A.; Ziaie, B. Laser-treated hydrophobic paper: An inexpensive microfluidic platform. Lab Chip 2011, 11, 1161-1165.

28. Niino, H.; Yabe, A. Surface modification and metallization of fluorocarbon polymers by excimer laser processing. Appl. Phys. Lett. 1993, 63, 3527-3529.

29. Jiang, L.; Sangeeth, C.S.S.; Yuan, L.; Thompson, D.; Nijhuis, C.A. One-nanometer thin monolayers remove the deleterious effect of substrate defects in molecular tunnel junctions. Nano Lett. 2015, 15, 6643-6649.

30. Nerngchamnong, N.; Wu, H.; Sotthewes, K.; Yuan, L.; Cao, L.; Roemer, M.; Lu, J.; Loh, K.P.; Troadec, C.; Zandvliet, H.J.W.; et al. The supramolecular structure of self-assembled monolayers of ferrocenyl terminated n-alkanethiolates on gold surfaces. Langmuir 2014, 30, 13447-13455

31. Nerngchamnong, N.; Yuan, L.; Qi, D.-C.; Li, J.; Thompson, D.; Nijhuis, C.A. Role of van der waals forces in performance of molecular diodes. Nature Nanotechnol. 2013, 8, 113-118.

32. Nijhuis, C.A.; Reus, W.F.; Siegel, A.C.; Whitesides, G.M. A molecular half-wave rectifier. J. Am. Chem. Soc. 2011, 133, 15397-15411.

33. Thuo, M.M.; Reus, W.F.; Nijhuis, C.A.; Barber, J.R.; Kim, C.; Schulz, M.D.; Whitesides, G.M. Odd-even effects in charge transport across self-assembled monolayers. J. Am. Chem. Soc. 2011, 133, 2962-2975.

34. Baghbanzadeh, M.; Simeone, F.C.; Bowers, C.M.; Liao, K.-C.; Thuo, M.; Baghbanzadeh, M.; Miller, M.S.; Carmichael, T.B.; Whitesides, G.M. Odd-even effects in charge transport across n-alkanethiolate-based SAMs. J. Am. Chem. Soc. 2014, 136, 16919-16925.

35. Thuo, M.M.; Reus, W.F.; Simeone, F.C.; Kim, C.; Schulz, M.D.; Yoon, H.J.; Whitesides, G.M. Replacing $-\mathrm{CH}_{2} \mathrm{CH}_{2}-$ with $-\mathrm{CONH}-$ Does Not Significantly Change Rates of Charge Transport through $\mathrm{Ag}^{\mathrm{TS}}-\mathrm{SAM} / / \mathrm{Ga}_{2} \mathrm{O}_{3} /$ EGaIn Junctions. J. Am. Chem. Soc. 2012, 134, 10876-10884.

36. Glavan, A.C.; Martinez, R.V.; Subramaniam, A.B.; Yoon, H.J.; Nunes, R.M.D.; Lange, H.; Thuo, M.M.; Whitesides, G.M. Omniphobic " $\mathrm{R}^{\mathrm{F}}$ paper" produced by silanization of paper with fluoroalkyltrichlorosilanes. Adv. Funct. Mater. 2014, 24, 60-70.

37. Oyola-Reynoso, S.; Heim, A.P.; Halbertsma-Black, J.; Zhao, C.; Tevis, I.D.; Cinar, S.; Cademartiri, R.; Liu, X.; Bloch, J.-F.; Thuo, M.M. Draw your assay: Fabrication of low-cost paper-based diagnostic and multi-well test zones by drawing on a paper. Talanta 2015, 144, 289-293.

38. Tevis, I.D.; Newcomb, L.B.; Thuo, M. Synthesis of liquid core-shell particles and solid patchy multicomponent particles by shearing liquids into complex particles (SLICE). Langmuir 2014, 30, 14308-14313.

39. Bel-Hassen, R.; Boufi, S.; Salon, M.-C.B.; Abdelmouleh, M.; Belgacem, M.N. Adsorption of silane onto cellulose fibers. II. The effect of $\mathrm{pH}$ on silane hydrolysis, condensation, and adsorption behavior. J. Appl. Polym. Sci. 2008, 108, 1958-1968.

40. Pallandre, A.; Glinel, K.; Jonas, A.M.; Nysten, B. Binary nanopatterned surfaces prepared from silane monolayers. Nano Lett. 2004, 4, 365-371. 
41. Mandal, S.; Das, G.; Dhar, S.; Tomy, R.M.; Mukhopadhyay, S.; Banerjee, C.; Barua, A.K. Development of a novel fluorinated n-nc-SiO:H material for solar cell application. Mater. Chem. Phys. 2015, 157, 130-137.

42. Bisanda, E.T.N.; Ansell, M.P. The effect of silane treatment on the mechanical and physical-properties of sisal-epoxy composites. Compos. Sci. Technol. 1991, 41, 165-178.

43. Hsieh, H.-Y.; Wang, P.-C.; Wu, C.-L.; Huang, C.-W.; Chieng, C.-C.; Tseng, F.-G. Effective enhancement of fluorescence detection efficiency in protein microarray assays: Application of a highly fluorinated organosilane as the blocking agent on the background surface by a facile vapor-phase deposition process. Anal. Chem. 2009, 81, 7908-7916.

44. Zhou, H.; Wang, H.; Niu, H.; Gestos, A.; Wang, X.; Lin, T. Fluoroalkyl silane modified silicone rubber/nanoparticle composite: A super durable, robust superhydrophobic fabric coating. Adv. Mater. 2012, 24, 2409-2412.

45. Zhang, C.; Shephard, N.E.; Rhodes, S.M.; Chen, Z. Headgroup effect on silane structures at buried polymer/silane and polymer/polymer interfaces and their relations to adhesion. Langmuir 2012, 28, 6052-6059.

46. Fang, L.; Chang, L.; Guo, W.-J.; Chen, Y.; Wang, Z. Influence of silane surface modification of veneer on interfacial adhesion of wood-plastic plywood. Appl. Surf. Sci. 2014, 288, 682-689.

47. Abdelmouleh, M.; Boufi, S.; Belgacem, M.N.; Duarte, A.P.; Ben Salah, A.; Gandini, A. Modification of cellulosic fibres with functionalised silanes: Development of surface properties. Int. J. Adhes. Adhes. 2004, 24, 43-54.

48. Valentini, L.; Macan, J.; Armentano, I.; Mengoni, F.; Kenny, J.M. Modification of fluorinated single-walled carbon nanotubes with aminosilane molecules. Carbon 2006, 44, 2196-2201.

49. Hayn, R.A.; Owens, J.R.; Boyer, S.A.; McDonald, R.S.; Lee, H.J. Preparation of highly hydrophobic and oleophobic textile surfaces using microwave-promoted silane coupling. J. Mater. Sci. 2011, 46, 2503-2509.

50. Karakoy, M.; Gultepe, E.; Pandey, S.; Khashab, M.A.; Gracias, D.H. Silane surface modification for improved bioadhesion of esophageal stents. Appl. Surf. Sci. 2014, 311, 684-689.

51. Bloodworth, R.; Penners, G.; Podszun, W.; Reiners, J.; Schulze, H. Silane-Modified Ethers or Polyethers; Stable Emulsifiers for Textiles, Metal Working; Mixtures. U.S. Patent US5171476, 15 December 1992.

52. Matienzo, L.J.; Egitto, F.D.; Logan, P.E. The use of silane coupling agents in the design of electrically stable interfaces of 6061 T6 aluminum alloy surfaces and epoxy-based electrically conductive adhesives. J. Mater. Sci. 2003, 38, 4831-4842.

53. Thickett, S.C.; Neto, C.; Harris, A.T. Biomimetic surface coatings for atmospheric water capture prepared by dewetting of polymer films. Adv. Mater. 2011, 23, 3718-3722.

54. O'Brien, E.P.; White, C.C.; Vogt, B.D. Correlating interfacial moisture content and adhesive fracture energy of polymer coatings on different surfaces. Adv. Eng. Mater. 2006, 8, 114-118.

55. Li, X.-W.; Zhang, Q.-X.; Guo, Z.; Yu, J.-G.; Tang, M.-K.; Huang, X.-J. Low-cost and large-scale fabrication of a superhydrophobic 5052 aluminum alloy surface with enhanced corrosion resistance. RSC Adv. 2015, 5, 29639-29646. 
56. Schiros, T.; Takahashi, O.; Andersson, K.J.; Ostrom, H.; Pettersson, L.G.M.; Nilsson, A.; Ogasawara, H. The role of substrate electrons in the wetting of a metal surface. J. Chem. Phys. 2010, 132.

57. Zhai, L.; Berg, M.C.; Cebeci, F.C.; Kim, Y.; Milwid, J.M.; Rubner, M.F.; Cohen, R.E. Patterned superhydrophobic surfaces: Toward a synthetic mimic of the namib desert beetle. Nano Lett. 2006, 6, 1213-1217.

58. Genzer, J.; Efimenko, K. Recent developments in superhydrophobic surfaces and their relevance to marine fouling: A review. Biofouling 2006, 22, 339-360.

59. Zhai, L.; Cebeci, F.Ç.; Cohen, R.E.; Rubner, M.F. Stable superhydrophobic coatings from polyelectrolyte multilayers. Nano Lett. 2004, 4, 1349-1353.

60. Andriot, M.; Chao, S.H.; Colas, A.; Cray, S.; de Buyl, F.; DeGroot, J.V.; Dupont, A.; Easton, T.; Garaud, J.L.; Gerlach, E.; et al. Silicones in Industrial Applications. In Inorganic Polymers; Jaeger, R.D., Gleria, M., Ed.; Nova Science Publishers: New York, NY, USA, 2007

61. Samyn, P. Wetting and hydrophobic modification of cellulose surfaces for paper applications. J. Mater. Sci. 2013, 48, 6455-6498.

62. Wong, T.-S.; Kang, S.H.; Tang, S.K.Y.; Smythe, E.J.; Hatton, B.D.; Grinthal, A.; Aizenberg, J. Bioinspired self-repairing slippery surfaces with pressure-stable omniphobicity. Nature 2011, 477, 443-447.

63. Wong, Y.; Yuen, C.; Leung, M.; Ku, S.; Lam, H. Selected applications of nanotechnology in textiles. AUTEX Res. J. 2006, 6, 1-8.

64. Chen, X.; Li, C.; Shao, W.; Du, H.; Burnell-Gray, J. The anti-static poly (ethylene terephthalate) nanocomposite fiber by in situ polymerization: The thermo-mechanical and electrical properties. J. Appl. Polym. Sci. 2007, 105, 1490-1495.

65. Yu, M.; Gu, G.; Meng, W.-D.; Qing, F.-L. Superhydrophobic cotton fabric coating based on a complex layer of silica nanoparticles and perfluorooctylated quaternary ammonium silane coupling agent. Appl. Surf. Sci. 2007, 253, 3669-3673.

66. Dastjerdi, R.; Montazer, M.; Shahsavan, S. A new method to stabilize nanoparticles on textile surfaces. Colloids Surf. A. 2009, 345, 202-210.

67. Hashem, M.; Ibrahim, N.A.; El-Shafei, A.; Refaie, R.; Hauser, P. An eco-friendly-novel approach for attaining wrinkle-free/soft-hand cotton fabric. Carbohydr. Polym. 2009, 78, 690-703.

68. Wang, Z. Nanolayer Self-Assembly on Ionic Fibers. Ph.D. Thesis, NC State University, Raleigh, NC, USA, 2009.

69. Dastjerdi, R.; Montazer, M. A review on the application of inorganic nano-structured materials in the modification of textiles: Focus on anti-microbial properties. Colloids Surf. B 2010, 79, 5-18.

70. Wang, Z.; Hauser, P.J.; Rojas, O.J. Study on charge distribution of carboxymethylated cotton fabric by streaming potential/current measurements. AATCC J. Res. 2015, 2, 13-19.

71. Hauser, P.J. Reducing pollution and energy requirements in cotton dyeing. Text. Chem. Color. Am. Dyest. Report. 2000, 32, 44-48.

72. Wang, Z.; Hauser, P.J.; Laine, J.; Rojas, O.J. Multilayers of low charge density polyelectrolytes on thin films of carboxymethylated and cationic cellulose. J. Adhes. Sci. Technol. 2011, 25, 643-660. 
73. Zhou, Z.; Liu, X.; Hu, B.; Wang, J.; Xin, D.; Wang, Z.; Qiu, Y. Hydrophobic surface modification of ramie fibers with ethanol pretreatment and atmospheric pressure plasma treatment. Surf. Coat. Technol. 2011, 205, 4205-4210.

74. Norton, F.J. Waterproofing Treatment of Materials. U.S. Patent US2386259 A, 9 October 1945.

75. Matuana, L.; Balatinecz, J.; Park, C.; Sodhi, R. X-ray photoelectron spectroscopy study of silanetreated newsprint-fibers. Wood Sci. Technol. 1999, 33, 259-270.

76. Zhu, Q.; Gao, Q.; Guo, Y.; Yang, C.Q.; Shen, L. Modified silica sol coatings for highly hydrophobic cotton and polyester fabrics using a one-step procedure. Ind. Eng. Chem. Res. 2011, 50, 5881-5888.

77. Shirgholami, M.A.; Khalil-Abad, M.S.; Khajavi, R.; Yazdanshenas, M.E. Fabrication of superhydrophobic polymethylsilsesquioxane nanostructures on cotton textiles by a solution-immersion process. J. Colloid Interface Sci. 2011, 359, 530-535.

78. Oyola-Reynoso, S.; Chen, J.; Cinar, S.; Bloch, J.F.; Thuo, M.M. Application of surface-adsorbed water as a co-monomer to create micro- and nano-sized particles in amphiphobic materials fabrication. J. Am. Chem. Soc. submitted.

79. Brazel, C.S.; Rosen, S.L. Fundamental Principles of Polymeric Materials; Wiley: Hoboken, NJ, USA, 2012.

80. Chen, S.; Li, L.; Zhao, C.; Zheng, J. Surface hydration: Principles and applications toward low-fouling/nonfouling biomaterials. Polymer 2010, 51, 5283-5293.

81. Çınar, S.; Anderson, D.D.; Akinc, M. Combined effect of fructose and $\mathrm{NaCl}$ on the viscosity of alumina nanopowder suspensions. J. Eur. Ceram. Soc. 2015, 35, 377-382.

82. Israelachvili, J.; Wennerstrom, H. Role of hydration and water structure in biological and colloidal interactions. Nature 1996, 379, 219-225.

83. Lefèvre, G.; Duc, M.; Lepeut, P.; Caplain, R.; Fédoroff, M. Hydration of $\gamma$-alumina in water and its effects on surface reactivity. Langmuir 2002, 18, 7530-7537.

84. Leng, C.; Han, X.; Shao, Q.; Zhu, Y.; Li, Y.; Jiang, S.; Chen, Z. In situ probing of the surface hydration of zwitterionic polymer brushes: Structural and environmental effects. J. Phys. Chem. C 2014, 118, 15840-15845.

85. Li, L.; Ren, L.; Wang, L.; Liu, S.; Zhang, Y.; Tang, L.; Wang, Y. Effect of water state and polymer chain motion on the mechanical properties of a bacterial cellulose and polyvinyl alcohol (BC/PVA) hydrogel. RSC Adv. 2015, 5, 25525-25531.

86. Rubasinghege, G.; Grassian, V.H. Role(s) of adsorbed water in the surface chemistry of environmental interfaces. Chem. Commun. 2013, 49, 3071-3094.

87. Fraxedas, J. Water at Interfaces: A Molecular Approach; CRC Press: Boca Raton, FL, USA, 2014.

88. Israelachvili, J.N. Intermolecular and Surface Forces, 3rd ed.; Academic Press: Saint Louis, MO, USA, 2011; p. 706.

89. Michot, L.J.; Villiéras, F.; François, M.; Bihannic, I.; Pelletier, M.; Cases, J.M. Water organisation at the solid-aqueous solution interface. Comptes Rendus Geosci. 2002, 334, 611-631.

90. Argyris, D.; Ashby, P.D.; Striolo, A. Structure and orientation of interfacial water determine atomic force microscopy results: Insights from molecular dynamics simulations. ACS Nano 2011, 5, 2215-2223. 
91. Ping, Z.H.; Nguyen, Q.T.; Chen, S.M.; Zhou, J.Q.; Ding, Y.D. States of water in different hydrophilic polymers-DSC and FTIR studies. Polymer 2001, 42, 8461-8467.

92. Tanaka, M.; Hayashi, T.; Morita, S. The roles of water molecules at the biointerface of medical polymers. Polym. J. 2013, 45, 701-710.

93. Verdaguer, A.; Sacha, G.M.; Bluhm, H.; Salmeron, M. Molecular structure of water at interfaces: Wetting at the nanometer scale. Chem. Rev. 2006, 106, 1478-1510.

94. Bullard, J.W.; Jennings, H.M.; Livingston, R.A.; Nonat, A.; Scherer, G.W.; Schweitzer, J.S.; Scrivener, K.L.; Thomas, J.J. Mechanisms of cement hydration. Cem. Concr. Res. 2011, 41, 1208-1223.

95. Marie-Pierre, G.; Michiel, S.; Marialore, S. Oxide/water interfaces: How the surface chemistry modifies interfacial water properties. J. Phys. Condens. Matter. 2012, 24, 124106.

96. Schönhoff, M.; Ball, V.; Bausch, A.R.; Dejugnat, C.; Delorme, N.; Glinel, K.; Klitzing, R.V.; Steitz, R. Hydration and internal properties of polyelectrolyte multilayers. Colloids Surf. A. 2007, $303,14-29$.

97. Gontard, N.; Guilbert, S.; Cuq, J.-L. Water and glycerol as plasticizers affect mechanical and water vapor barrier properties of an edible wheat gluten film. J. Food Sci. 1993, 58, 206-211.

98. Mali, S.; Sakanaka, L.S.; Yamashita, F.; Grossmann, M.V.E. Water sorption and mechanical properties of cassava starch films and their relation to plasticizing effect. Carbohydr. Polym. 2005, 60, 283-289.

99. Oyola-Reynoso, S.; Tevis, I.D.; Chen, J.; Bloch, J.-F.; Thuo, M.M. Fabrication of namib beetle inspired biomimetic amphiphobic surfaces using adsorbed water as a co-monomer. Procedia Eng. 2016, accepted.

100. Song, J.; Rojas, O.J. Approaching super-hydrophobicity from cellulosic materials: A review. Nord. Pulp Pap. Res. J. 2013, 28, 216-238.

101. Nishino, T.; Meguro, M.; Nakamae, K.; Matsushita, M.; Ueda, Y. The lowest surface free energy based on $-\mathrm{CF}_{3}$ alignment. Langmuir 1999, 15, 4321-4323.

(C) 2015 by the authors; licensee MDPI, Basel, Switzerland. This article is an open access article distributed under the terms and conditions of the Creative Commons Attribution license (http://creativecommons.org/licenses/by/4.0/). 